\title{
Das SIWF: Der Zukunft der ärztlichen Bildung verpflichtet
}

Der vorliegende Geschäftsbericht informiert in Zahlen, Worten und Schemata über die Tätigkeiten des Schweizerischen Instituts für ärztliche Weiter- und Fortbildung im vergangenen Jahr. Er vermittelt Einblicke in das breite Tätigkeitsspektrum des Instituts und im Rahmen eines Interviews in die Themen, welche die Geschäftsleitung besonders beschäftigen.

Eine Sorge bereitet uns die Entwicklung der Rahmenbedingungen für die Weiterbildung an den Spitälern, wo den betriebswirtschaftlichen Aspekten mehr und mehr oberste Priorität eingeräumt wird. Es bedarf des Engagements aller Verantwortlichen, die Bedürfnisse der Weiterbildung wirkungsvoll zu vertreten, denn die Vermittlung der notwendigen Kompetenzen an die kommende Ärztegeneration ist essenziell für das Gesundheitswesen der Zukunft.

Zunehmend im Blickpunkt auch von aussen steht die Fortbildung als Pflicht für alle klinisch tätigen Ärztinnen und Ärzte. Sie basiert auf der Selbstverantwortung eines akademischen Berufes, muss aber glaubhaft dokumentiert werden und muss den Erhalt und heute auch die Weiterentwicklung der beruflichen Kompe- tenz sicherstellen. Dafür stellt das SIWF die Fortbildungsplattform zur Verfügung, die es erlaubt, die notwendigen Fortbildungs-Credits unkompliziert zu registrieren und sich nach Erfüllen der Anforderungen das Fortbildungsdiplom ausdrucken zu lassen.

$\mathrm{Zu}$ den vielen Aktivitäten des SIWF gehören auch die speditive und sorgfältige Erledigung der vielen Anträge auf Anerkennung von Weiterbildungsstätten sowie der Titelgesuche nach absolvierter Facharztweiterbildung, das Organisieren von Tagungen, Sitzungen und über hundert Visitationen sowie die Beantwortung einer stets steigenden Zahl von Anfragen. Für ihre kompetente und engagierte Arbeit möchten wir allen Mitarbeiterinnen und Mitarbeitern, aber auch der Geschäftsleitung, dem Vorstand und allen Kommissionsmitgliedern herzlich danken.

Weiterhin gilt uneingeschränkt, was schon einmal die Überschrift eines Vorwortes zum Geschäftsbericht war: effizient administrieren und stetig weiterentwickeln.

\section{Bildnachweis}

Tobias Schmid/SIWF

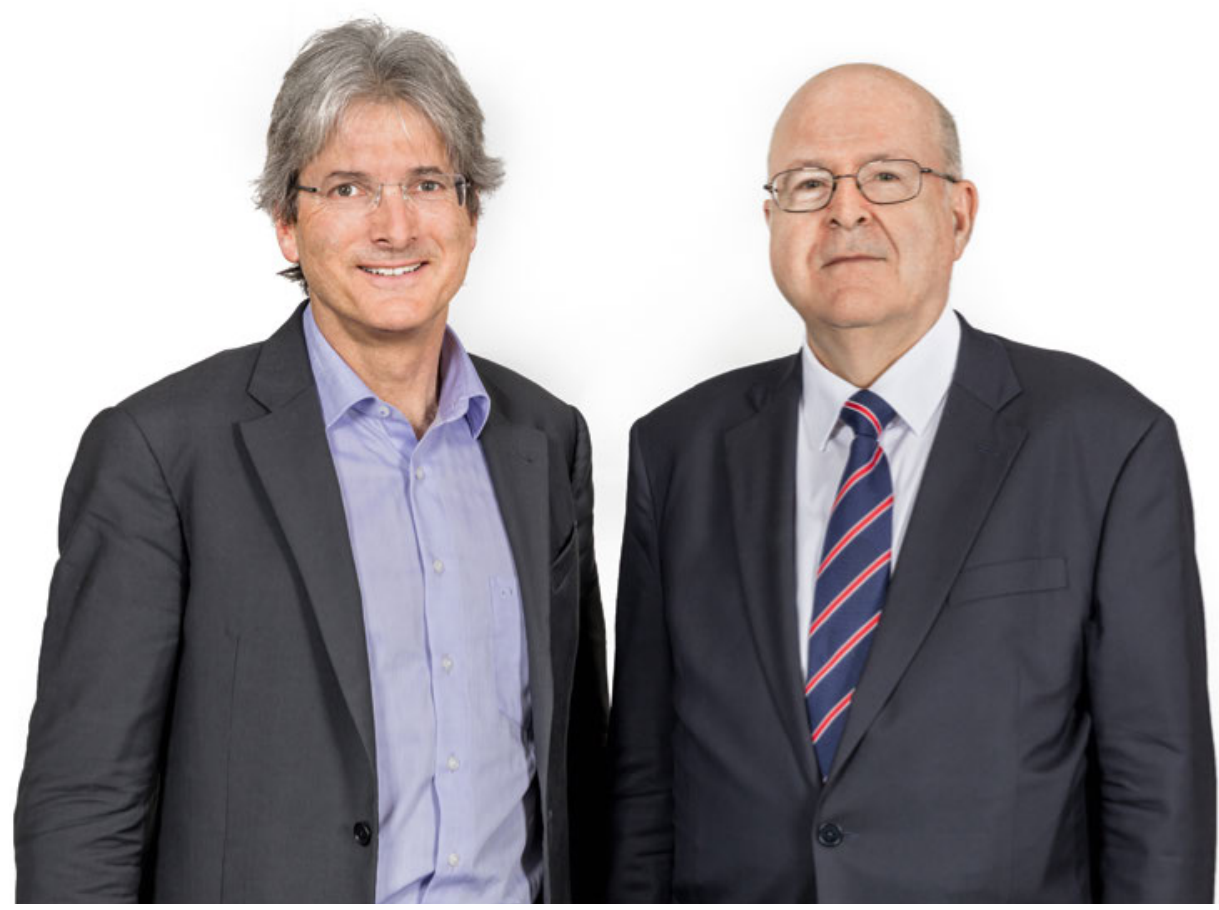

Christoph Hänggeli, Rechtsanwalt, Geschäftsführer SIWF und Dr. med. Werner Bauer, Präsident SIWF 


\section{$\underset{\substack{\text { SIWFM } \\ \text { SWEM }}}{\operatorname{SW}} \mid 2017$}
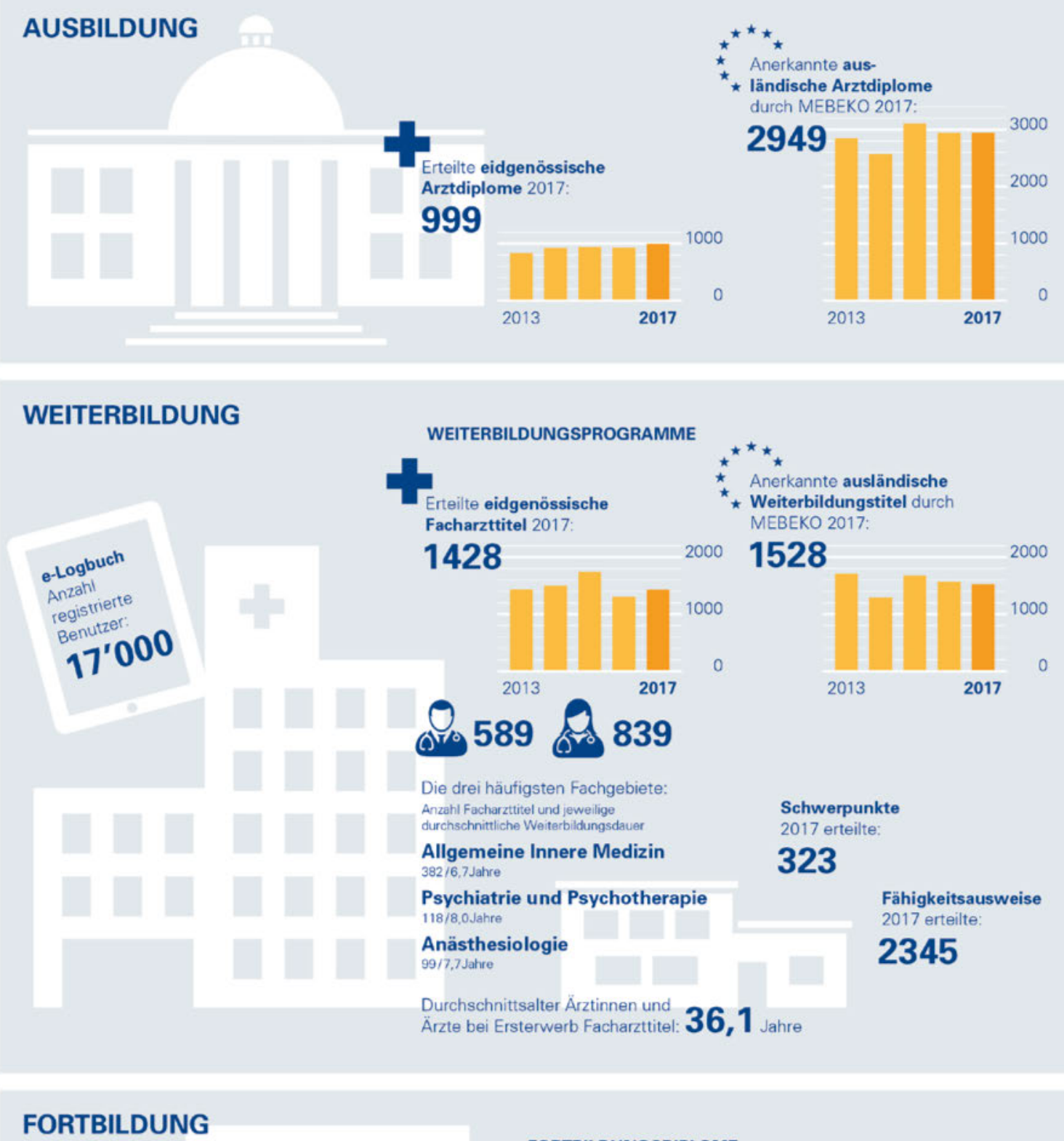

\section{FORTBILDUNGSDIPLOME}

\begin{tabular}{|c|c|c|}
\hline $\begin{array}{l}\text { Selber erfasste } \\
\text { via e-Fortbildungs- } \\
\text { plattform: }\end{array}$ & $\begin{array}{l}\text { Von den Fach- } \\
\text { gesellschaften } \\
\text { gemeldete: }\end{array}$ & $\begin{array}{l}\text { Ärztinnen und Ärzte } \\
\text { mit Fortbildungs- } \\
\text { diplom insgesamt: }\end{array}$ \\
\hline 4812 & 570 & \\
\hline
\end{tabular}

Anzahl anerkannte $\begin{gathered}\text { SIWF } \\ \text { ISFM }\end{gathered}$ 


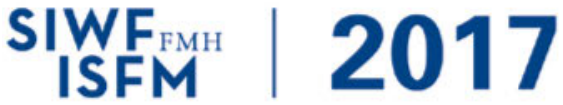

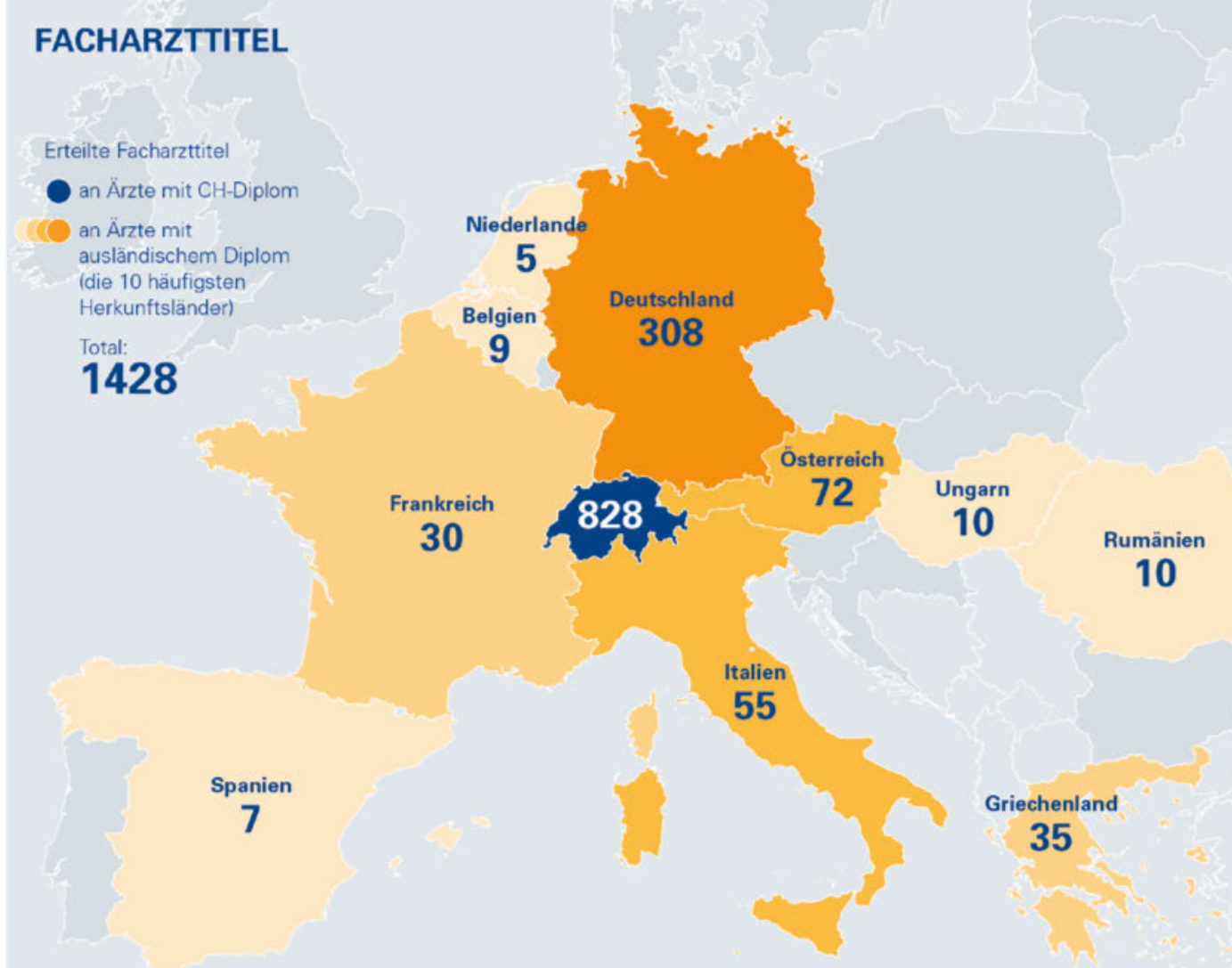

ASSISTENZ-

ÄRZTE-

UMFRAGE
An Umfrage teilgenommen:

8060

Assistenzärztinnen und -ärzte

(Rücklaufquote 69,8\%)

\section{3}

ausgewertete

Weiterbildungsstätten

82 von 100 Weiterbildung und Privatleben zufrieden bis sehr zufrieden.

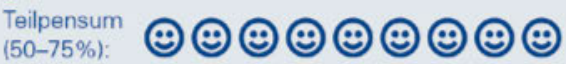

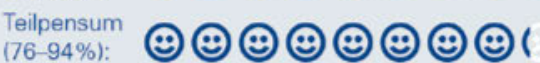

Vollzeitstelle $(100 \%)$

Die Entlastung bei den administrativen Tätigkeiten sehen Assistenzärztinnen und -ärzte als wirksamste Massnahme zur Verbesserung der Vereinbarkeit von Weiterbildung und

Korrespondenz:

Schweizerisches Institut

für ärztliche Weiter-

und Fortbildung SIWF

FMH

Elfenstrasse 18

CH-3000 Bern 15

Tel. 031359111

siwf[at]fmh.ch

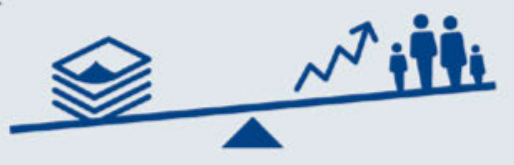

Soll gezielt gesteuert werden, wie viele Arzte/Arztinnen pro Fachrichtung weitergebildet werden?

Assistenzärzte/ Leiter/innen -ärztinnen: der WBS:
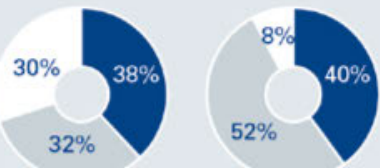

Ja Nein Weiss nicht 